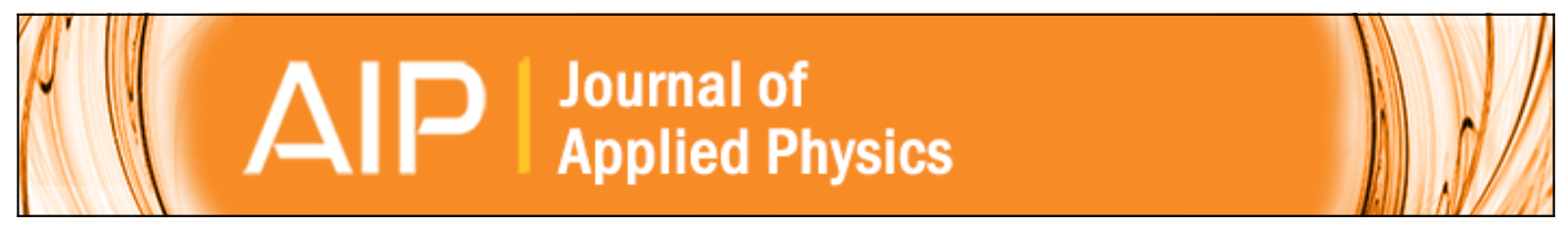

\title{
Computer simulation of electron beams. I. Space-charge algorithm for asymmetric
} beams

D. A. de Wolf

Citation: Journal of Applied Physics 58, 3692 (1985); doi: 10.1063/1.335630

View online: http://dx.doi.org/10.1063/1.335630

View Table of Contents: http://scitation.aip.org/content/aip/journal/jap/58/10?ver=pdfcov

Published by the AIP Publishing

\section{Articles you may be interested in}

Electron-beam diagnostic for space-charge measurement of an ion beam

Rev. Sci. Instrum. 76, 023301 (2005); 10.1063/1.1847392

Simulation of transverse combining of space-charge dominated beams

AIP Conf. Proc. 152, 278 (1986); 10.1063/1.36336

Simulation of space-charge limiting current in relativistic electron beams

Phys. Fluids 22, 799 (1979); 10.1063/1.862632

Decay of Space-Charge Waves on Electron Beams

J. Appl. Phys. 37, 1904 (1966); 10.1063/1.1708623

Crossed Electron Beam Technique for Measuring Space-Charge Effects in Beams

J. Appl. Phys. 25, 679 (1954); 10.1063/1.1721715

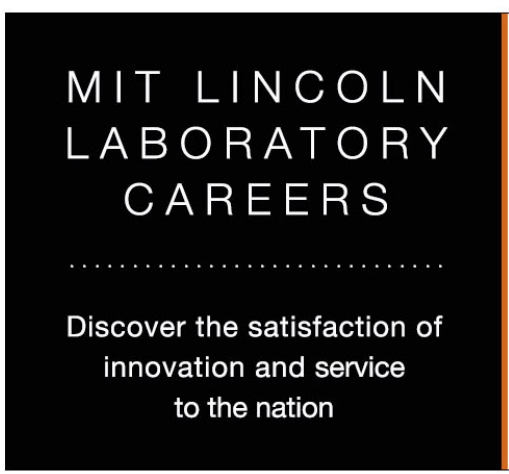
- Space Control
- Air \& Missile Defense
- Communications Systems
\& Cyber Security
- Intelligence, Surveillance and
Reconnaissance Systems

Electronics

- Tactical Systems
- Homeland
Protection
- Air Traffic Control

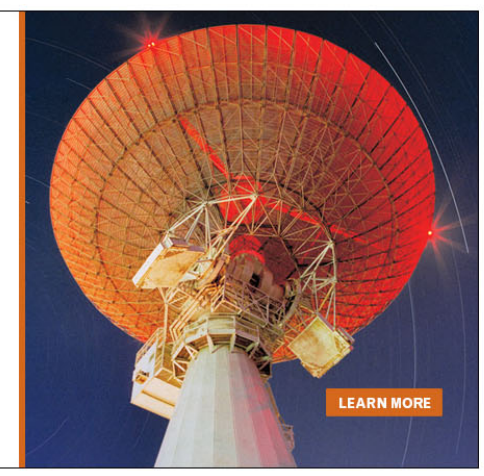




\title{
Computer simulation of electron beams. I. Space-charge algorithm for asymmetric beams
}

\author{
D. A. de Wolfa) \\ Department of Electrical Engineering, Virginia Polytechnic Institute and State University, Blacksburg. \\ Vinginia 24061
}

(Received \July 1985; accepted for publication 1 August 1985)

\begin{abstract}
Longitudinal space-charge forces can be neglected in computer simulations of slowly curving quasilaminar electron beams. A particle-mesh-type algorithm for transverse space-charge forces is developed, and great simplification in numerical calculation of asymmetric electron beams is demonstrated. Some typical examples for kinescope beams are discussed.
\end{abstract}

\section{IATRODUCTION}

Accurate manipulation of electron beams is the key to design of many electron-optics devices such as CRT tubes, accelerators, etc. The advent of the digital computer has made it possible to calculate the trajectories of key electrons in the beam under the influence of the electromagnetic fields created inside the device by external sources. The inclusion, however, of the mutual Coulomb-repulsion forces of the electrons (space charge) makes the calculation nonlinear and therefore greatly increases the calculation time and expense. The numerical solution of the ensuing trajectory equations then requires a number of iterations; each iteration solves a trajectory equation with space-charge forces inferred from the solution of the previous iteration. Suppose $N$ electron trajectories are to be calculated numerically, and each trajectory requires $L / \Delta z=l$ steps. In the absence of space-charge forces, $\mathrm{Nl}$ fields and potentials must be calculated in total (each field has three components, moreover). The inclusion of space-charge forces increases this number of fields and potentials to $M N^{2} l$ (where $M$ is the number of iterations). Aside from the extra factor $M N$, the calculation is also made more difficult by the need to avoid $1 / r$-type singularities in the Coulomb forces.

We show that it is possible to ignore the longitudinal space-charge force component for thin electron beams. This removes the need for iteration; i.e., reduces the space-charge calculation by a factor $M$. Also, the relative smoothness of transverse space-charge forces (with respect to interparticle distances) makes it possible to formulate a "particle-mesh force" algorithm of the type defined by Hockney and Eastwood. The factor $N^{2}$ is then reduced to $n N$, where $n$ is a mesh number much less than $N$. The algorithm is particularly useful for intricate "pencil" beams, e.g., as in kinescopes where lensing action mixes an initial thermal distribution of electrons into a long, quasilaminar ${ }^{2}$ beam of electron trajectories. The beam may curve slowly with respect to its diameter; however, its perveance should not become too large (see Appendix A). Most important, no symmetry properties are assumed.

\section{NEGLECT OF LONGATUDINAL SPACE-CHARGE FORCES}

Let the beam be characterized by a smooth charge density $\rho(r)$ which is not a function of time. The nonrelativistic

\footnotetext{
This work was performed while the author was still at RCA Laboratories, Princeton, NJ 08540.
}

equations of motion are, for $\mathbf{r}=\left(\mathbf{r}_{T}, z\right)$

$$
\begin{aligned}
& \ddot{\mathbf{r}}=-(e / m)\left[\mathbb{E}\left(\mathbf{r}_{T}, z\right)+\dot{\mathbf{r}} \times \mathbf{B}_{0}\left(\mathbf{r}_{T}, z\right)\right], \\
& D H_{1}(z) / D z=0,
\end{aligned}
$$

where $H_{1}(z)=m\left(\dot{z}^{2}+\dot{\mathbf{r}}_{T}^{2}\right) / 2-e \Phi\left(\mathbf{r}_{T}, z\right)$ is the single-particle Hamiltonian, $e$ is the electron charge, $\Phi$ is the electrostatic potential, $\mathbf{E}=-\nabla \phi$ is the electrostatic field, and $\mathbf{B}_{0}$ is an externally applied magnetic field (the small induced field at $r$ due to other particle motion is neglected). The second equation expresses conservation of $H_{1}(z)$ along a trajectory. The time dependence can be replaced by dependence on $z$ (denoted by primes) through

$$
\begin{aligned}
& \dot{\mathbf{r}}_{T}=\dot{z} \mathbf{r}_{T}^{\prime}, \\
& \ddot{\mathbf{r}}_{T}=\dot{z}^{2} \mathbf{r}_{T}^{\prime \prime}+\frac{1}{2}\left(d \dot{z}^{2} / d z\right) \mathbf{r}_{T}^{\prime},
\end{aligned}
$$

and $\dot{z}^{2}$ can be replaced by potentials, using $H_{1}(z)=H_{1}(0)$. The problem lies in the fact that the field $E\left(r_{T}, z\right)$ is a solution of the Poisson equation,

$$
\boldsymbol{\nabla} \cdot \mathbf{E}(\mathbf{r})=\epsilon_{0}^{-1} \rho(\mathbf{r}),
$$

where $\epsilon_{0}$ is the vacuum dielectric permittivity. However, $\rho(\mathbf{r})$ is determined by the trajectories of the electrons so that Eqs. (1) and (3) are coupled when space-charge forces are not negligible. The usual method of solution is iterative; the $n$th trajectory calculations use the charge density inferred from the $(n-1)$ st trajectories.

For the applications under consideration, an electron beam changes direction slowly, i.e., the local radius of curvature is large compared to the local diameter. The location $r$ in the beam can be decomposed into a set of locally orthogonal coordinates $\mathbf{r}=(\xi, \xi)$, where $\hat{\xi}$ is a unit vector in the main axial direction and $\xi_{x}, \hat{\xi}_{y}$ are two others in a plane normal to $\hat{\zeta}$. The transverse space-charge approximation rests on the assumption that $\rho(\xi, \zeta)$ depends only weakly upon $\zeta$. In general, however, the space-charge field $\mathbb{E}_{\mathrm{sp}}(\mathbf{r})$ can be obtained from the integral form of Eq. (3),

$$
\mathbb{E}_{\mathrm{sp}}(\mathbf{r})=\frac{1}{4 \pi \epsilon_{0}} \int \mathrm{d}^{3} \mathrm{r}_{1} \rho\left(\mathbf{r}_{1}\right) \frac{\mathbf{r}-\mathbf{r}_{1}}{\left|\mathbf{r}-\mathbf{r}_{1}\right|^{3}},
$$

but when $\partial \rho / \partial \zeta_{1}=0$, this simplified to the area integral over a cross section of the bearn at $\xi_{1}=\xi$ :

$$
E_{\mathrm{sp}}(\xi, \xi)=\frac{1}{2 \pi \epsilon_{0}} \int \mathrm{d}^{2} \xi \rho(\xi, \xi) \frac{\xi-\xi_{1}}{\left|\xi-\xi_{1}\right|^{2}} .
$$

This removes the iterative coupling because $\mathbb{E}_{\mathrm{sp}}(\mathbf{r})$ then de- 
pends only upon the past history of the trajectories so that the step from $\mathbf{r}_{T}(z)$ to $\mathbf{r}_{T}(z+\Delta z)$ does not require anything other than knowledge of local initial conditions. Equations (1), (2), and (5) can then be solved in a noniterative straightforward numerical procedure.

The approximation $\partial \rho / \partial \xi_{1}=0$ in Eq. (4) does require that trajectory directions do not differ drastically from $\hat{\zeta}_{1}$, and also that acceleration not be very large along the beam. The magnitude of the space-charge forces also play a role because these cause the electrons to diverge from each other, which affects $\rho\left(\xi, \zeta_{2}\right)$ for $\xi_{2}>\zeta_{1}$. A criterion is given in Appendix $A$ for the restriction upon space-charge forces. These restrictions are not serious in kinescope drift regions, nor in lensing regions where the acceleration along the beam direction is slow over a length of several diameters, and where beam perveance is small.

\section{盺. DEVELOPABENT OF TME ALGORITHM}

The elimination of time from Eqs. (1) and (2) transforms the trajectory equations into a set of ordinary second-order differential equations in $z$ with initial conditions, if approximation (5) is valid. Whether linear multistep or single-step Runge-Kutta methods ${ }^{3}$ are used, we can illustrate what is involved by considering an equivalent set of difference equations:

$$
\begin{aligned}
& \mathbf{r}_{i}(z+\Delta z)=\mathbf{r}_{i}(z)+\mathbf{r}_{i}^{\prime}(z) \Delta z+\frac{1}{2} \mathbf{r}_{i}^{\prime \prime}(z) \Delta z^{2}, \\
& \mathbf{r}_{i}^{\prime}(z+\Delta z)=\mathbf{r}_{i}^{\prime}(z)+\mathbf{r}_{i}^{\prime \prime}(z) \Delta z, \\
& \mathbf{r}_{i}^{\prime \prime}(z)=f\left[\mathbb{E}\left[\mathbf{r}_{i}(z)\right], \mathbb{B}_{0}, \mathbf{r}_{i}^{\prime}\right\} .
\end{aligned}
$$

The functional depencience of $r_{i}^{\prime \prime}(z)$ need not be specified in detail here; it follows from Ecss. (1), (2), and (5). In particleparticle ${ }^{4}$ methods, the beam is represented by $N$ trajectories, each with characteristic charge $q,(1<j<N)$. The spacecharge part of the field $\mathbb{E}\left[\mathbf{r}_{i}(z)\right]$ acting upon particle $i$ can then be written as

$$
\begin{aligned}
\mathbb{E}_{s p}^{(i)} & \equiv \mathbb{E}_{s p}\left[\mathbf{r}_{i}(z)\right] \\
& =\frac{1}{2 \pi \epsilon_{0}} \sum_{j=1}^{N} q_{j} \frac{\xi_{i}-\xi_{j}}{\left|\xi_{i}-\xi_{j}\right|^{2}} \equiv \sum_{j=1}^{N} \mathbb{E}_{i j}
\end{aligned}
$$

in terms of the locally orthogonal locations $\xi_{j}$ of the $j$ th trajectory from trajectory $i$. The $j=i$ term must be excluded, and $q_{j}$ is conserved along a representative trajectory. The solution of Eq. (6) for one step $\Delta z$ requires $\frac{1}{2} N(N-1)$ calculations of $\mathrm{E}_{i j}$, hence roughly $N^{2} L / 2 \Delta z$ calculations (where $L$ is the total path length) are needed. This poses two problems: not only is $N^{2}$ a large number, but also $N$ would have to be chosen extremely large in order to ensure that near singularities for $\xi_{j}$ close to $\xi_{i}$ are balanced out by other near trajectories.

Particle-mesh methods ${ }^{5}$ offer an attractive alternative. Consider for illustrative purposes only that the beam travels in the $z$ direction and let $\mathbf{r}=(x, y, 0)$ be the transverse coordinate. Define a square mesh in a plane normal to $z$, and let $M$ mesh squares cover the cross section of the beam. Define a mesh density

$$
\rho_{j}=\frac{1}{h^{2}} \sum_{i=1}^{N} q_{i} \theta\left[\mathbf{r}_{i}(z)-\mathbf{R}_{j}(z)\right],
$$

where $\mathbb{R}_{j}(z)$ is the center of the $j$ th mesh square with area $h^{2}$, and $\theta\left(\mathbf{r}_{1}-\mathbf{r}_{2}\right)$ is zero when $\left|x_{1}-x_{2}\right|>h / 2$ or $\left|y_{1}-y_{2}\right|$ $>h / 2$, and unity otherwise. The new densities should be smoothly varying, and each mesh square should contain many trajectories. Using Eq. (5), one finds for the spacecharge fields at $r$ due to a square of area $h^{2}$ centered at $r_{j}$ with uniform charge density $\rho_{j}$

$$
\begin{aligned}
& E_{x}(\mathbf{r})= K\left[\frac{1}{2} t_{f} \ln \left(\frac{t_{f}^{2}+s_{f}^{2}}{t_{i}^{2}+s_{f}^{2}}\right)-\frac{1}{2} t_{i} \ln \left(\frac{t_{f}^{2}+s_{i}^{2}}{t_{i}^{2}+s_{i}^{2}}\right)\right. \\
&\left.+2 s_{f}\left[\arctan \left(t_{f} / s_{f}\right)-\arctan \left(t_{i} / s_{f}\right)\right]-2 s_{i}\left[\arctan \left(t_{f} / s_{i}\right)-\arctan \left(t_{i} / s_{i}\right)\right]\right], \\
& s_{f}=x-x_{j}+\frac{1}{2} h, \quad t_{f}=y-y_{j}+\frac{1}{2} h, \quad s_{i}=x-x_{j}-\frac{1}{2} h, \quad t_{i}=y-y_{j}-\frac{1}{2} h, \quad K=h^{2} \rho_{j} / 2 \pi \epsilon_{0} .
\end{aligned}
$$

A similar expression holds for $E_{y}(\mathbf{r})$. Equation (9) suggests the following interpolation formulas for $\mathbb{E}_{\mathrm{sp}}(\mathbf{r}, \boldsymbol{z})$ :

$$
W_{\mathrm{ap}}[\mathbf{r}(z)]=K \sum_{j=1}^{M} \frac{\mathbf{r}-\mathbf{r}_{j}(z)}{\left|\mathbf{r}-\mathbf{r}_{j}(z)\right|^{2}+h^{2} / \pi} .
$$

Each term of $E q .(10)$ has the same limiting value as $r \rightarrow r_{j}(z)$ or as $r \rightarrow \infty$ as the corresponding form of Eq. (9); the computational advantage of Eq. (10) over Eq. (9) consists of not needing to evaluate a number of logarithms and arctangents for each term. The major discrepancies between Eqs. (10) and (9) occur at distances $|\mathbf{r}-\mathbf{r}(z)| \sim \frac{1}{2} h$, as illustrated in Fig. 1 which plots the ratio of the $x$ component of Eq. (9) to that of one term of Eq. (10) for $K=1, h=1$. Therefore, Eq. (10) will converge towards the correct answer as $h \rightarrow 0$ providing $h$ does not become so small that the graininess of chosen elec- tron trajectories becomes a factor (see Appendix B).

\section{SOME NUMERICAL RESULTS}

The simplest space-charge problem is the expansion of an axially symmetric uniform laminar beam containing current $I$ and with initial waist radius $R_{0}{ }^{6}$ The radius at distance $z, R(z)$, is given analytically by Eq. (A2), and the expansion factor $r(z) / r(0)$ of any core of initial width $r(0)$ is a constant at fixed z. A test case was chosen in a $3.5 \mathrm{~mA}$ beam of initial radius $R_{0}=50$ (in arbitrary units). The electron velocity was chosen to be equivalent to $30 \mathrm{keV}$. At $z=13500$ units, Eqs. (A2) yield an expansion factor 1.3522 (to a four-decimal-place accuracy). The numerical results for $\gamma(z)$ are given in Fig. 2 as a function of $\gamma(z)=1.3522 r(0)$. The beam was discretized into 16 circle sectors, each of which 


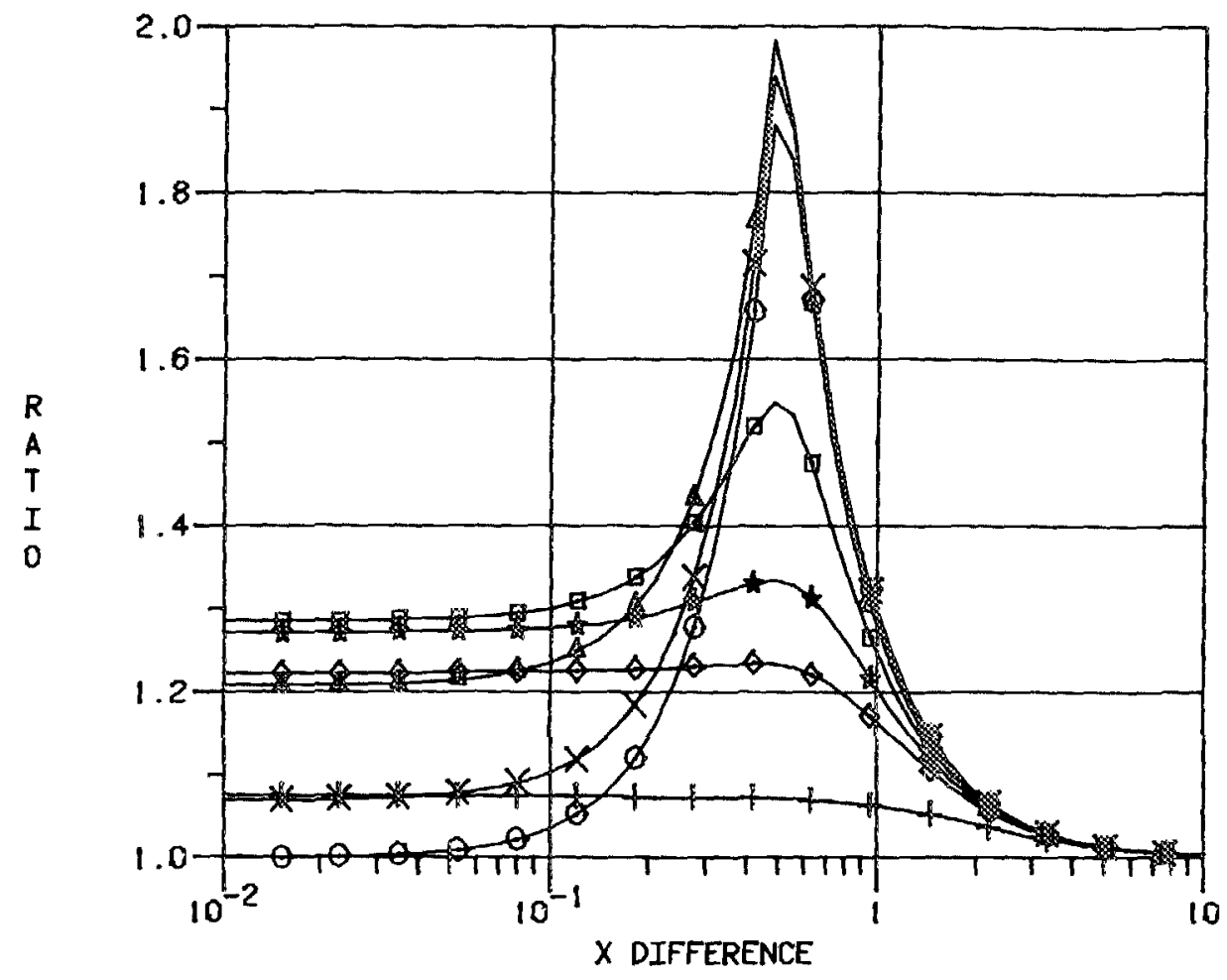

FIG. 1. Ratio of exact to approximated $E_{x}$ space-charge field (in a plane) from a uniform unit square vs $x$ distance from its center for $y$ distance $0(0), 0.2(\times), 0.4(\triangle), 0.6$ (D), $0.8\left(^{*}\right), 1.0(0)$, and $2.0(1)$. was divided into 18 annular pieces of equal radial width. The space-charge mesh consisted of 169 squares. While that choice means only a few electrons per mesh square, the regularity of the discretization avoids the pitfalls of granularity, and Fig. 2 shows a good representation of the laminar beam in only five steps in the $z$ direction. Figure 3 shows predictions of several electron landing positions in a typical kinescope drift-region beam discretized into $1500-1600$ representative trajectories. These data are for a $3.5 \mathrm{~mA}$ beam with velocity of the electrons very close to $25 \mathrm{keV}$, and the drift distance is 13.5 in. along the axis. Radial symmetry has been

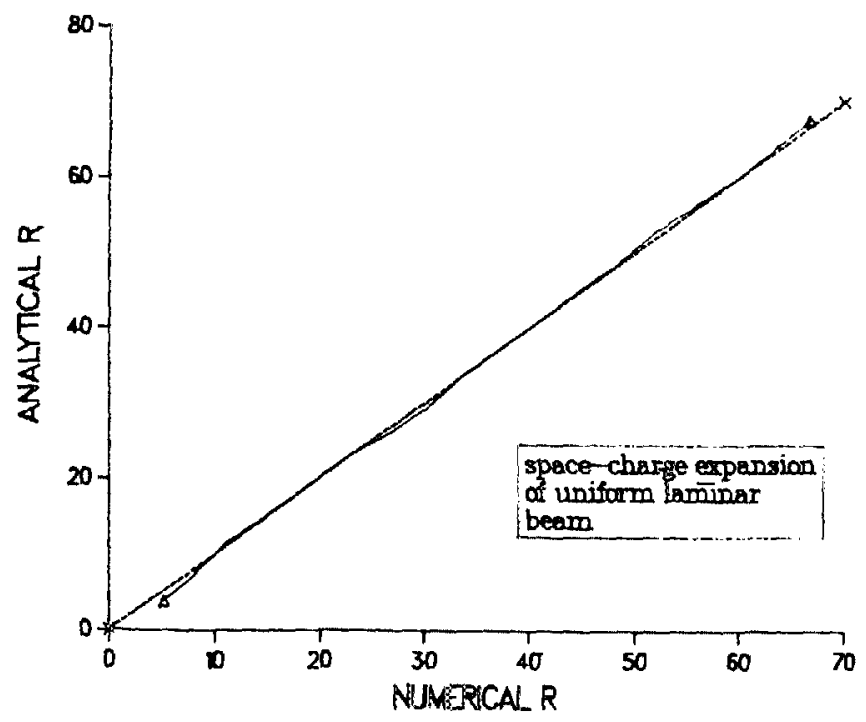

FIG. 2. Comparison of space-charge expansion of a uniform laminar beam under the transverse-force algorithm to the analytical result (ordinate) for expansion constant $\approx 1.3522$. The full line would coincide with the $45^{\circ}$ line if there were perfect agreement. chosen for simplicity, although no use has been made of it. An initial phase space (consisting of values of $x, x^{\prime}, y, y^{\prime}$, and charge density $q$ for each beamlet) is prepared at the entrance to the drift region. This phase space is quite distorted from a typical input cathode phase space because the electrons have passed through a number of electrostatic lenses and have also undergone aberrations. Moreover, the input phase space at the cathode is complicated by a thermal distribution of emitted electrons. The details are discussed elsewhere. ${ }^{8}$ Figure 3 compares the unapproximated numerical calculation based upon Eq. (4) with an axisymmetric space-charge



FIG. 3. Comparison $r=d r / d z$ vs $r$ of selected kinescope electrons after a drift of $13.5^{\prime \prime}$ at $25 \mathrm{keV}$ for various values of the number of mesh squares NMESH ( $1 \mathrm{mil}=0.001 \mathrm{in}$ ). The ELOP values are calculated from an unapproximated Poisson equation. 
program ELOP' to diverse caĺculations based upon Eq. (5) with the algorithm of this paper. Various choices of the number of mesh squares are shown. The smoothness of the spacecharge forces is apparent in this type of beam because the results for $\mathrm{NMESH}=121$ (121 squares) hardly differ from those for NMESH = 25 (25 squares). Moreover, there is good agreement everywhere with the ELOP calculation, except for a very weak edge beamlet that is highly aberrated. Further comparisons entail calculations of beam current densities, but these are discussed in a companion paper ${ }^{10}$ as the problem of beam representation is a topic in its own right.

A typical simulation of 1519 trajectories over $11 z$ steps with a space-charge grid of 49 squares required $18 \mathrm{CPU}$ seconds on an IBM 3081 computer. We estimate that a full. scale three-dimensional space-charge calculation in a similar particle-particle simulation would require of the order of $3000 \mathrm{cpu}$ seconds on the same computer, ${ }^{10}$ unless simplified analytical calculations are considered. ${ }^{11-13}$ Hence it appears that reasonably accurate simulations of electron beams at low cost are quite feasible with the algorithm. ${ }^{14}$

\section{APPENDIX A: NEGLECT OF LONGTTUDINAL SPACE- CHARGE FIELD}

In order to study restrictions upon the approximation of Eq. (4) by Eq. (5), consider an axisymmetric laminar beam of transversely uniform density $\rho(z)$ with a waist of radius $R_{0}$ at $z=0$. Then the $x$ component of the space-charge field, according to Eq. (4), is

$$
\begin{aligned}
E_{x}(\mathbf{r})= & \frac{1}{4 \pi \epsilon_{0}} \int d^{2} r_{1} \int_{-\infty}^{\infty} d z_{1} \rho\left(z_{1}\right) \\
& \times \frac{x-x_{1}}{\left[\left(\mathbf{r}-\mathbf{r}_{1}\right)^{2}+\left(z-z_{1}\right)^{2}\right]^{3 / 2}}
\end{aligned}
$$

By using $\rho\left(z_{1}\right)=j\left(z_{1}\right) / \dot{z}_{1}=(m / 2 e)^{1 / 2} j\left(z_{1}\right) / \Phi^{1 / 2}$ under the assumption that $\Phi$ is the velocity in $\mathrm{eV}$ and $j\left(z_{1}\right)$ is the current density in the $z_{1}$ plane, we note that the insertion of Eq. (Al) into Eq. (1), for $B_{0}=0$, leads to the well-known ${ }^{15}$ expression for the beam radius $R_{0}(z)$ at $z$ :

$$
\begin{aligned}
& R_{0}(z) \equiv R_{0} \exp W^{2}, \\
& \int_{0}^{W} d t e^{t^{2}}=K_{p}^{1 / 2} z / R_{0}, \\
& K_{p} \equiv \beta I / 4 \Phi^{3 / 2}, \quad \beta \equiv(m / 2 e)^{1 / 2} / 2 \pi \epsilon_{0},
\end{aligned}
$$

where $I=\pi R_{0}^{2} j(0)$ is the total current, and $K_{p}$ is the perveance (a well-known measure of space-charge force in a beam). An important feature of Eq. (A2), and of Gauss' law, is that a laterally uniform beam at $z=0$ remains so at other values of $z$. Now apply Eq. (A1) at $z=0, r=\mathbb{R}_{0}$ (i.e., at the edge of the beam):

$$
\begin{aligned}
E_{x} \approx & \frac{1}{2 \pi \epsilon_{0}} \int d^{2} r_{1} \frac{x-x_{1}}{\left|\mathbf{R}_{0}-\mathbf{r}_{1}\right|^{2}} \\
& \times\left(\frac{1}{2} \int_{-L / 2}^{L / 2} d z_{1} \rho\left(z_{1}\right) \frac{\left|\mathbf{R}_{1}-\mathbf{r}_{1}\right|^{2}}{\left[\left|\mathbf{R}_{0}-\mathbf{r}_{1}\right|^{2}+z^{2}\right]^{3 / 2}}\right) .
\end{aligned}
$$

The truncation of the $d z$ integral at $\pm L / 2$ is not a serious error as long as $L>2 R_{0}$. However, for weak perveance $K_{p}$ so that $K_{p} z^{2} / R_{0}^{2}<1$, Eq. (A2) is well approximated by $R_{0}(z)=R_{0}+K_{p} z^{2} / R_{0}$, and it is quite obvious that $z=L / 2$ can be quite large in the above sense if $K_{p}$ is sufficiently small. From this and from the homogeneity of the beam at all $z$ it follows that to first order, for $|z| \leqslant L / 2$,

$$
\rho(z) \approx\left(1+K_{p} z^{2} / R_{0}^{2}\right)^{-2} \rho(0) .
$$

Let the new variable $t=z /\left|\mathbf{R}_{0}-\mathbf{r}_{1}\right|$ in Eq. (A3) and insert Eq. (A4). We obtain

$$
\begin{aligned}
& E_{x}=\frac{\rho(0)}{2 \pi \epsilon_{0}} \int d^{2} r_{1} \frac{x-x_{1}}{\left|\mathbf{R}_{0}-\mathbf{r}_{1}\right|^{2}} G\left[K_{p}\left|\mathbf{R}_{0}-\mathbf{r}_{1}\right|^{2} / R_{0}^{2}\right] \\
& G(Q) \approx \int_{0}^{\infty} d t\left(1+Q t^{2}\right)^{-2}\left(1+t^{2}\right)^{-3 / 2}
\end{aligned}
$$

and $Q$ is an abbreviation for the product $K_{p}\left|R_{0}-r_{1}\right|^{2} / R_{0}^{2}$. The extension of the upper bound of the integral to infinity is no problem due to the rapid falloff of the integrand with $t$. The $G(Q)$ integral can be given in closed form,

$$
G(Q)=\frac{1+Q / 2}{(1-Q)^{2}}+\frac{Q(1-Q / 4)}{(1-Q)^{5 / 2}} \ln \left(\frac{1-(1-Q)^{1 / 2}}{1+(1-Q)^{1 / 2}}\right),
$$

which can be expanded for small $Q$ into the series,

$$
G(Q)=1+Q \ln Q+(3 / 2+\ln 4) Q+O\left(Q^{2} / n Q\right)+\cdots \text {. }
$$

Hence $G(Q)=1$ is a good approximation for $Q<1$, and the error is of order $Q \ln Q$. Because $Q \leq K_{p}$ it follows that the error in setting $G(Q)=1$ is of order $K_{p} \ln K_{p}$, and also that $E_{x}$ is approximated well by Eq. (5). So even in the most favorable case of a laminar beam, there is a restriction on beam perveance that limits the validity of neglecting longitudinal space-charge forces.

\section{APPENDIX B: ACCURACY OF THE SPACE-CHARGE FIELD ESTHMATE}

The use of Eq. (10) instead of Eq. (5) involves several approximations. These are examined here in some more detail. Consider a mesh square of area $S=h^{2}$ in the cross section of the beam and assume $z$ is the beam axis of importance for simplicity. The integral of importance is

$$
E_{x}(\mathbf{r})=\int_{s} d^{2} r_{1} \rho\left(\mathbf{r}_{1}\right) G_{x}\left(\mathbf{r}-\mathbf{r}_{1}\right)
$$

where $G_{x}\left(r-r_{1}\right)=\left(x-x_{1}\right) /\left|r-r_{1}\right|^{2}$, and $\mathbf{r}$ is the point where space-charge due to the square is measured. Define

$$
\langle\rho\rangle \equiv \frac{1}{h^{2}} \int_{S} d^{2} r_{1} \rho\left(\mathbf{r}_{1}\right), \quad Q(\mathbf{r}) \equiv \frac{1}{h^{2}} \int_{S} d^{2} r_{1} G_{x}\left(\mathbf{r}-\mathbf{r}_{1}\right),
$$

and let $\bar{Q}(\mathbf{r}) \equiv\left(x-x_{1}\right) /\left[\left|\mathbf{r}-\mathbf{r}_{1}\right|^{2}+h^{2} / \pi\right]$. It is easily seen that (B1) can be rewritten as

$$
\begin{aligned}
E_{x}(\mathbf{r})= & \langle\rho\rangle \bar{Q}(\mathbf{r})+h^{2}(\rho\rangle[Q(\mathbf{r})-\bar{Q}(\mathbf{r})] \\
& +\int_{S} d^{2} r_{1}\left[\rho\left(\mathbf{r}_{1}\right)-\langle\rho\rangle\right] G_{x}\left(\mathbf{r}-\mathbf{r}_{1}\right)
\end{aligned}
$$

The first term is the approximation Eq. (10), and the remaining terms are the error estimates. Figure 1 shows that the second term is negligible, except for an area around 
$\left|\mathbf{r}-\mathbf{r}_{1}\right| \sim h / 2$ that decreases in proportion to the rest as $h \rightarrow 0$. The third term also vanishes as $h \rightarrow 0$ provided the mesh square contains many trajectories so that $\langle\rho\rangle$ is smoothly varying from the one square to the next.

'R. W. Hockney and J. W. Eastwood, Computer Simulation Using Particles (McGraw-Hill, New York, 1981).

${ }^{2}$ By "quasilaminar," we mean beams that do allow for crossing trajectories, but in which the effective beam envelope (containing, e.g., 90\% of the current) does not change in diameter rapidly over many diameters length.

${ }^{3}$ E. g. J. D. Lambert, in The State of the Ant in Numerical Analysis, edited by D. Jacobs (Academic, London, 1977), p. 451.

${ }^{\prime}$ R. W. Hockney and J. W. Eastwood, Computer Simulation Using Particles (McGraw-Hill, New York, 1981), p. 18. See also T. Groves, D. L. Hammond, and H. Kuo, J. Vac. Sci. Technol, 16, 1680 (1979).
${ }^{5}$ R. W. Hockney and J. W. Eastwood, Computer Simulation Using Particles (McGraw-Hill, New York, 1981), p. 120.

${ }^{6} \mathrm{P}$. T. Kirsten, G. S. Kino, and W. E. Waters, Space-Charge Flow (McGraw-Hill, New York, 1967).

'A. L. Lichtenberg, Phase-Space Dynamics of Particles (Wiley, New York, 1969).

${ }^{8}$ R. C. Alig, RCA Rev. 41, 517 (1980).

'F. J. Campbell (private communication).

${ }^{10}$ D. A. de Wolf, J. Appl. Phys. 58, 3697 (1985).

"W. P. Lysenko, IEEE Trans. Nucl. Sci. NS-26, 3508 (1979).

${ }^{12}$ W. P. Lysenko and E. A. Wadlinger, IEEE Trans. Nucl. Sci. NS-28, 2509 (1981).

${ }^{13} \mathrm{~W}$. J. Gallagher, IEEE Trans. Nucl. Sci. NS-28, 2552 (1981).

${ }^{14}$ The estimate is based largely upon the considerations of Sec. I, and partly upon the ELOP program which exploits axial symmetry and is therefore much more efficient than an equivalent asymmetric computer program.

${ }^{15}$ O. Klemperer (and M. E. Barnett, in collaboration), Electron Optics, 3rd edition (Cambridge University, London, 1971). 